\title{
Predictive value of hyperglycosylated human chorionic gonadotropin for pregnancy outcomes in threatened abortion in first-trimester viable pregnancies
}

\author{
Valor predictivo de la hCG hiperglosilada para predecir resultado \\ de la gestación en amenaza de aborto de primer trimestre en \\ gestación viable
}

https://doi.org/10.23938/ASSN.933

\author{
A. Salas ${ }^{1}$, B. Gastón ${ }^{2}$, J. Barrenetxea ${ }^{2}$, T. Sendino ${ }^{3}$, M. Jurado ${ }^{1}$, J.L. Alcázar ${ }^{1}$
}

\begin{abstract}
Background. To investigate the value of a single determination of hyperglycosylated hCG (hCG-H) for predicting the clinical outcome of patients with threatened abortion in the first trimester of pregnancy.

Methods. Prospective study performed on 86 consecutively selected women with a diagnosis of threatened abortion and viable intrauterine pregnancy in the first trimester of pregnancy, conducted in two tertiary care hospitals. All patients underwent a single blood sample to determine hCG-H and total hCG serum levels and a transvaginal ultrasound 12-24 hours after diagnosis. Patients were monitored to determine whether the outcome was a miscarriage before the 20th week of pregnancy.
\end{abstract}

Results. Forty-three women (50\%) had a miscarriage during the follow-up. We observed a very high correlation between hCG-H and total hCG $(r=0.91, \mathrm{p}<0.001)$. Median hCG-H and total hCG from pregnancies with normal outcome was significantly higher than those ending in abortion. hCG-H and total hCG were very similar predictors of pregnancy outcomes (AUC: 0.90 and 0.89 , respectively). The ratio hCG-H / total hCG was a poor predictor (AUC: $0.64)$.

Conclusion. A single hCG-H assay is helpful for predicting pregnancy outcomes in women with first trimester threatened abortion and viable or potentially viable pregnancy at the time of presentation. However, hCG-H is not a better predictor than total hCG.

Keywords. hCG. Early pregnancy. First trimester bleeding. Threatened abortion. Miscarriage.

\begin{abstract}
RESUMEN
Fundamento. Investigar el valor de una única determinación de hCG hiperglicosilada (hCG-H) para predecir el resultado clínico de pacientes con amenaza de aborto en el primer trimestre del embarazo.
\end{abstract}

Métodos. Estudio prospectivo realizado en 86 mujeres, seleccionadas consecutivamente, con diagnóstico de amenaza de aborto y embarazo intrauterino viable en el primer trimestre de embarazo, realizado en dos hospitales de tercer nivel. A todas las pacientes se les realizó una única extracción sanguínea para determinar los niveles séricos de hCG-H y hCG total, y una ecografía transvaginal 12-24 horas después del episodio de sangrado. Se realizó seguimiento de las pacientes para determinar si el resultado fue un aborto espontáneo antes de la semana 20 de embarazo.

Resultados. Cuarenta y tres mujeres (50\%) sufrieron un aborto espontáneo durante el seguimiento. Se observó una correlación muy alta entre hCG-H y hCG total $(\mathrm{r}=0,91$, $\mathrm{p}<0,001)$. La mediana de hCG-H y hCG total de los embarazos con resultado normal fue significativamente mayor que la de aquellos que terminaron en aborto. La hCG-H y la hCG total fueron predictores muy similares del resultado del embarazo (AUC: 0,90 y 0,89, respectivamente). La relación hCG-H / hCG total fue un mal predictor (AUC: 0,64).

Conclusión. La determinación única de hCG-H es útil para predecir el resultado del embarazo en mujeres con amenaza de aborto en el primer trimestre y embarazo viable en el momento de la presentación clínica. Sin embargo, la hCG-H no es mejor predictor que la hCG total.

Palabras clave. hCG. Gestación precoz. Sangrado en el primer trimestre. Amenaza de aborto. Aborto espontáneo.

\section{An. Sist. Sanit. Navar. 2021; 44 (1): 23-31}

1. Departamento de Obstetricia y Ginecología. Clínica Universidad de Navarra. Pamplona. España

2. Departamento de Obstetricia y Ginecología. Complejo Hospitalario de Navarra. Pamplona. España.

3. Laboratorio de Bioquímica. Clínica Universidad de Navarra. Pamplona. España.

Received: October 26, 2020

Revised: December 9, 2020

Accepted: December 29, 2020

\author{
Corresponding author: \\ Juan Luis Alcázar \\ Departamento de Obstetricia y Ginecología \\ Clínica Universidad de Navarra \\ Avda. Pío XII, 36 \\ 31008 Pamplona \\ E-mail: jlalcazar@unav.es
}




\section{INTRODUCTION}

A miscarriage is a pregnancy that ends spontaneously before the fetus reaches a gestational age that enables it to be viable $^{1}$. The incidence of spontaneous miscarriages is estimated to be between $10-20 \%$ of all clinically recognized pregnancies ${ }^{1,2}$. However, the real incidence is very difficult to establish since those that occur very early generally go unnoticed ${ }^{1,2}$.

It has been estimated that $85 \%$ of spontaneous miscarriages occur in the first trimester $^{2,3}$. Early pregnancy loss that occurs in the first twelve weeks of gestation is defined as a nonviable intrauterine pregnancy, and clear sonographic criteria have been established for such a diagnosis in both symptomatic and asymptomatic women ${ }^{3,4}$.

The exact reason why miscarriage occurs is still a matter of debate and in many cases a single cause for this outcome remains unidentified. Some authors state that one third of miscarriages are due to chromosomal abnormalities and that two thirds of pregnancy failures are due to improper implantation of the embryo ${ }^{5}$. However, in very early pregnancy, chromosomal abnormalities are much more frequent ${ }^{6}$.

Threatened abortion is one of the most frequent clinical situations in Gynecology and Obstetrics emergency services, occurring in approximately $20-30 \%$ of pregnancies $^{2}$. Clinically speaking, threatened abortion is characterized by genital bleeding or hemorrhage of variable intensity from the uterine cavity, which may or may not be associated with mild-moderate pain in a woman with amenorrhea and a positive pregnancy test, with live or potentially alive fetus/embryo ${ }^{7}$. First trimester bleeding may be a marker of incorrect embryo implantation and incorrect placental development ${ }^{7}$.

Management of this clinical situation currently involves several medical visits before establishing the definitive diagnosis and prognosis, and this process entails a substantial consumption of health resources ${ }^{1}$. Currently, a correct clinical history, physical examination, transvaginal ultrasound and, if necessary, single or serial assays of $\beta$-hCG in blood are performed ${ }^{4}$. The $\beta$-hCG duplica- tion test requires two clinical visits in a very specific time window; in addition, this test has a limited sensitivity of $62 \%-78 \%$ and a false positive rate of 26 to $40 \%$

A variant form of hCG is the so-called hyperglycosylated hCG (hCG-H), which differs from hCG in terms of its site of synthesis, molecular structure, and function ${ }^{12-17}$. Hyperglycosylated hCG originates from placental extravillous cytotrophoblast cells in an autocrine manner ${ }^{18,19}$ and has a more complex structure than $\mathrm{hCG}^{20,21}$.

Hyperglycosylated hCG is the predominant isoform in the first weeks of gestation. It represents $80 \%$ of the hCG produced in the first week after implantation, it makes up $92 \%$ of the total hCG at three weeks of gestation and is gradually replaced by other isoforms ${ }^{22,23}$. The presence of hCG-H in early pregnancy is related to the process of implantation and invasiveness of trophoblast cells ${ }^{24,25}$. Hyperglycosylated hCG has been shown to antagonize receptor II of TGF beta, blocking cellular regulation, reducing apoptosis in cytotrophoblastic cells, promoting growth and invasion of cells in the cytotrophoblast $^{26,27}$. An imbalance in hCG-H values may be the cause of pregnancy failure, since some studies have shown that excessively low proportions of hCG-H are observed in miscarriage ${ }^{28}$. However, evidence about the role of hCG-H in predicting pregnancy outcomes in first-trimester threatened abortion is still scanty.

In this study we hypothesized that hCG-H can predict pregnancy outcomes in first-trimester threatened abortion viable pregnancies. We therefore set out to investigate the value of a single determination of hCG-H for predicting the clinical outcome of patients with threatened abortion in the first trimester of pregnancy.

\section{METHODS}

The study was prospective. The individuals selected as eligible for the research process were women with a diagnosis of threatened abortion in the first trimester of pregnancy (4-12 weeks) who attended the Emergency Room (ER) of the Clinica 
Universidad de Navarra (CUN) or the Complejo Hospitalario de Navarra (CHN) from June 2016 to March 2018. STROBE guidelines were followed.

Inclusion criteria were as follows: 1) patients with a positive urine pregnancy test at home with gestational amenorrhea $\leq 12$ weeks and with knowledge of the date of the last menstrual period; 2) spontaneous pregnancy; 3 ) viable pregnancies from detection of embryo heart activity, crumprump length $<7 \mathrm{~mm}$, visible heart activity was not required ${ }^{3,4}$; 4) clinically stable patients at the time of initial diagnosis without exacerbations at the clinic that forced us to perform urgent clinical interventions; 5) written informed consent provided.

Exclusion criteria were as follows: 1) patients who were diagnosed on the first ultrasound evaluation with a gestation of undetermined location or an ectopic gestation; 2) multiple gestation at diagnosis or detected at follow-up scan; 3) gestational trophoblastic disease; 4) diagnosis of aneuploidy; 5) assisted reproductive technique used as a conception method; 6) patients with a personal history of hypertension or diabetes mellitus.

The main outcome sought in the analysis was the occurrence of miscarriage before the twentieth week of gestation (yes/no).

All patients recruited were centralized at CUN, where the first assessment was carried out in the first 12-24 hours after diagnosis in the ER. The following variables were collected: patients' age (years), body mass index (BMI, $\mathrm{kg} / \mathrm{m}^{2}$ ), gestational age at presentation (days) according to last menstrual date, and previous pregnancies, deliveries and abortions. A single blood sample was drawn $(10 \mathrm{ml}$ of blood to obtain four aliquots) and stored at $-80^{\circ} \mathrm{C}$ at the Department of Biochemistry of the CUN, which kept the samples until the deferred determination of HCG and hCG-H (mUI / $\mathrm{mL}$ ). This determination was made once all the patients had been recruited. Total hCG levels along with the $\beta$ subunit were analyzed in all serum samples using an automated electrochemiluminescence immunoassay (Cobas 8000, Roche) and hCG-H using a commercial ELISA (MybioSource). For this analysis, the guidelines provided by the manufacturing laboratory were followed.

In addition, a first transvaginal ultrasound evaluation was performed, using two ultrasound machines equipped with a 5-9 $\mathrm{MHz}$ vaginal probe (Voluson E10 and E8, General Electric Healthcare, Milwaukee, WI, USA). The uterus and adnexa were examined and measured. Gestational sac diameters and volume were measured. The presence or absence of the yolk sac and/or embryo was determined. If the embryo was present, the crown-rump length (CRL) was measured and heartbeat was noted. If heartbeat was present or the embryo had a CRL $<7 \mathrm{~mm}$ but the heartbeat was not observed the patient was included. If the embryo's CRL was $\geq 7 \mathrm{~mm}$ and heartbeat was negative, miscarriage was confirmed and the patient was excluded. The gestational sac volume was estimated using the Volume Organ Computer Aided analysis (VOCAL ${ }^{\mathrm{TM}}$ ) method using the 4D-View software (General Electric Healthcare, Zipf, Austria) as per a previously described technique ${ }^{29}$. The same examiner (AS) performed all ultrasound examinations, measurements and volume calculations.

During this first visit, the patient received verbal and written information regarding all the pertinent aspects of the study. If she decided to enter the study, she had to sign an informed consent. Institutional Review Board approval was obtained before starting the study from both participating institutions (CUN and CHN).

The patients were reassessed clinically within two weeks after the diagnosis of threatened abortion. If the patient presented with heavy bleeding beforehand, she was reassessed earlier. In all cases, an ultrasound scan was performed to confirm the viability of pregnancy. From then on a weekly follow-up by telephone was also carried out until the twentieth week of gestation.

The patients were clinically managed by the relevant center (CUN or CHN) as per the local protocol. Clinicians managing the patients did not have information about the hCG- levels.

The clinical, laboratory and ultrasound data obtained from the patients were included in a database designed for this pur- 
pose. The Kolmogorov-Smirnov test was used to assess normal distribution of continuous data. Data are expressed as mean with standard deviation (SD) or median with interquartile range (IQR) depending on data distribution. The categorical variables were presented as absolute number and percentage.

Continuous variables were compared using the t-test or the Mann-Whitney test depending on the distribution. Categorical variables were compared using the chisquared test. The rho (r) Spearman correlation coefficient was used to analyze the correlation between hCG-H and total hCG and patient's age, gestational age and sac volume.

We carried out linear and quadratic regression analysis to establish the ratio of hCG-H and total hCG with gestational age in normal outcome. The best curve to fit was chosen according to the highest $\mathrm{R}^{2}$, all coefficients that were different from zero, and the least residuals. We then compared the data from patients with normal outcome and patients whose pregnancies ended in miscarriage with an analysis of covariance, taking into account the gestational age as a co-variable. We used logarithmic transformation of absolute values of hCG-H and total hCG.

Receiver operating characteristics (ROC) curves were plotted to determine the best total hCG and hCG-H cut-off for predicting pregnancy outcome. The corresponding area under the curve (AUC) of the ROC curve was estimated and compared using Hanley's method. We set out to determine whether the ratio of hCG-H/total hCG is a better predictor than a single H-hCG determination, as a number of authors have proposed $^{12}$. Therefore, the ROC curve for hCG-H/total hCG was also plotted and the AUC was estimated. A sub-analysis was performed, comparing the area under the curve of the ROC curve of total hCG, hCG-H and hCG-H/total hCG ratio in the women who had a living embryo with threatened abortion at the time of presentation.

All statistical analyses were carried out with the Statistical Package for the Social Sciences (SPSS) program, version 20.0.
(SPSS Inc.; Chicago, IL). A p value $<0.05$ as statistically significant was used in all tests.

\section{RESULTS}

Eighty-six patients were recruited during the study period, all with a diagnosis of threatened first trimester miscarriage and potentially viable or viable pregnancy. The outcome was obtained in all cases.

Forty-three women (50\%) had an ongoing normal pregnancy at the twentieth week of gestation and forty-three (50\%) ended in miscarriage before the twentieth week of gestation. Figure 1 shows the patients' flow chart.

Patients presenting with threatened abortion at the Emergency Room

$(n=313)$

Patients included $\mathrm{n}=86$

ART: assisted reproductive technique; PUL: pregnancy of unknown location.

Figure 1. Flow chart showing selected patients.

The mean age of the patients was 34 years (SD: 4.71), which was slightly higher in patients whose pregnancies ended in miscarriage (Table 1). The mean values of BMI, gestational age, number of previous gestations and previous abortions were similar between both groups of patients. Patients whose pregnancies ended in miscarriage had had less previous deliveries. 
Table 1. Demographic characteristics of the patients included in this study

\begin{tabular}{lccrlr}
\hline Characteristics & Global & Normal outcome & \multicolumn{2}{c}{ Miscarriage } & p \\
\hline Age (years) & $34(4.71)$ & $33.0(4.1)$ & 35.1 & $(5-1)$ & 0.046 \\
BMI (kg/m2) & $22.9(4.3)$ & $22.9(4.0)$ & 23.1 & $(4.5)$ & 0.786 \\
Gestational age (days) & $50.3(11.2)$ & $52.3(11.9)$ & 48.3 & $(10.3)$ & 0.103 \\
Number of previous pregnancies & $2.53(1.73)$ & $2.51(1.9)$ & 2.6 & $(1.6)$ & 0.902 \\
Number of previous deliveries* & $0.47(0.84)$ & $0.7(1.0)$ & 0.3 & $(0.5)$ & 0.037 \\
Number of pevious abortions & $0.88 \quad 1.15)$ & $0.7(0.9)$ & 1.0 & $(1.3)$ & 0.135 \\
\hline
\end{tabular}

All characteristics were expressed as mean (SD) except *: median (IQR). All p-values were obtained from t-test except $\uparrow$ : Mann Whitney U.

Median hCG-H and total hCG from the pregnancies with a normal outcome was significantly higher than the ones that ended in miscarriage (Table 2, Fig. 2).

Table 2. Biochemical data from the patients included in this study

\begin{tabular}{lrrr}
\hline \multicolumn{1}{c}{ Data } & Normal Outcome & Miscarriage & p \\
\hline Total hCH (UI/nL) & $62,929(65,498)$ & $2,850(13,576)$ & $<0.001$ \\
hCG-H (UI/mL) & $5,489.4(5,008.1)$ & $1,889.2(1,207.9)$ & $<0.001$ \\
Ratio hCG-H / hCG & $8.9(2.6)$ & $7.8(4.1)$ & $<0.001$ \\
\hline
\end{tabular}

All data were expressed as median (IQR); all p-values were obtained from Mann Whitney U test.
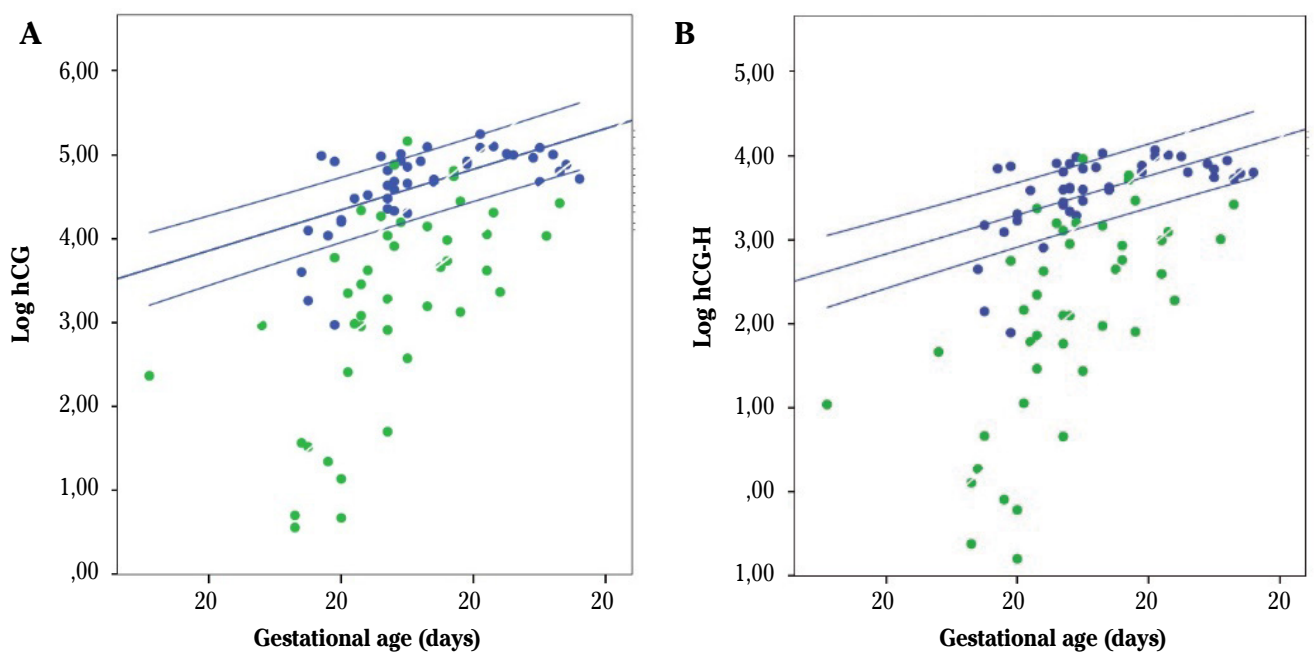

Figure 2. Log-transformed concentrations with 95\% confidence interval according to gestational age of the pregnancy in pregnancies that continued (blue dots) $\left(\mathrm{R}^{2}=0.356\right)$ and in pregnancies with miscarriage (green dots). A. Total hCG, $\mathrm{R}^{2}=0.356$. B. hCG-H, $\mathrm{R}^{2}=0.380$.

We observed a significant correlation between gestational age and hCG-H $(r=0.58$, $\mathrm{p}<0.001)$ and total hCG $(\mathrm{r}=0.62, \mathrm{p}<0.001)$. We also observed a very high correlation between hCG-H and total hCG $(\mathrm{r}=0.91$, $\mathrm{p}<0.001)$. We also found a significant correlation between gestational sac volume and $\mathrm{hCG}-\mathrm{H}(\mathrm{r}=0.69, \mathrm{p}<0.001)$ and total $\mathrm{hCG}$ 
$(\mathrm{r}=0.78, \mathrm{p}<0.001)$. However, we saw that there was no relationship between patient's age and hCG-H $(\mathrm{r}=-0.28, \mathrm{p}=0.72)$ or total hCG $(\mathrm{r}=-0.13, \mathrm{p}=0.42)$.

ROC curve analysis showed that the best cut-off of hCG-H and total hCG for predicting pregnancy outcome were $2,698 \mathrm{mIU} / \mathrm{mL}$ and $28,880 \mathrm{mIU} / \mathrm{mL}$, respectively. The AUCs for both parameters were very similar (AUC $=0.90,95 \% \mathrm{CI}: 0.84-0.97$ vs. $\mathrm{AUC}=0.89$, 95\%CI: 0.81-0.96; $\mathrm{p}=0.891$ ). The hCG-H/total hCG ratio was a poor predictor for pregnancy outcome (AUC $=0.64,95 \% \mathrm{CI}$ : 0-52-0.76) (Fig. 3A).
A

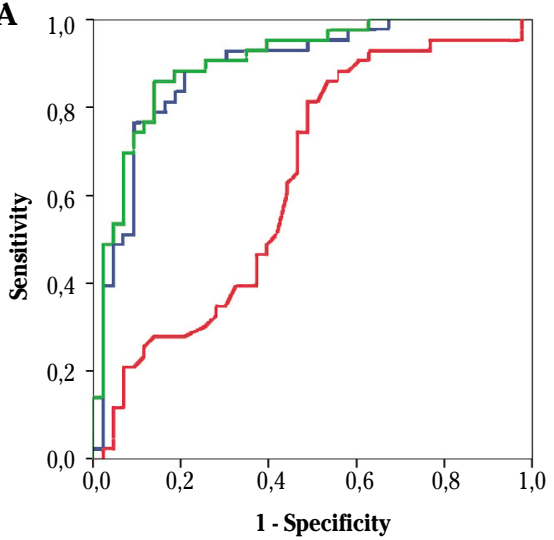

B

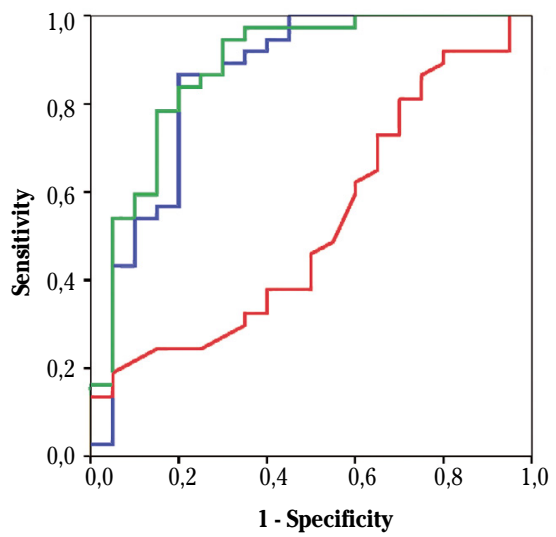

Figure 3. ROC curves for total hCG (blue), hCG-H (green) and ratio hCG-H/total hCG (red). A. All women in the study. B. Women with living embryo.

When we considered only women who had a living embryo when they presented with threatened abortion, we observed that AUC for hCG-H $(0.88,95 \%$ CI: 0.78 to 0.98$)$ and total hCG $(0.85,95 \% \mathrm{CI}: 0.73$ to 0.97$)$ were similar (Fig. 3B). Therefore, both parameters were predictors for pregnancy outcome with similar accuracy. The ratio hCG-H/total hCG was a poor predictor (AUC $=0.52,95 \% \mathrm{CI}: 0.36$ to 0.68$)$.

\section{DISCUSSION}

In this study, we investigated the predictive value of a single hCG-H serum determination in patients with first-trimester threatened abortion and viable pregnancy at the time of presentation. We have found that hCG-H may be useful in predicting a pregnancy outcome. However, we observed that hCG-H was not a better predictor than hCG. The patient's age was also related to the pregnancy outcome.
The main strength of our study is that, to the best of our knowledge, this is the first attempt to assess the role of hCG-H for predicting a pregnancy outcome in women with first-trimester threatened abortion and viable or potentially viable pregnancy. Some studies have reported on the role of hCG-H in predicting pregnancy outcome in early pregnancy, but not in the clinical scenario of threatened abortion.

Sutton-Riley et al published a study investigating whether hCG-H may predict gestation failure ${ }^{10}$. They assessed blood and urine samples from women in the first nine weeks of pregnancy and compared total hCG and hCG-H levels in normal and failing gestations (miscarriage and ectopic pregnancy). They found that hCG-H (threshold level of $13 \mu \mathrm{g} / \mathrm{L}$ ) concentration in blood samples had a sensitivity of $73 \%$ with a false positive rate of $2.9 \%$ for detecting failing pregnancies. Sensitivity for hCG, using the same false positive rate, was $42 \%$. For urine tests (using the same threshold 
level of $13 \mu \mathrm{g} / \mathrm{L}$ ), these figures had a sensitivity of $75 \%$ with a false positive rate of $15 \%$ for detecting failing pregnancies for hCG-H, whereas sensitivity for hCG, using the same false positive rate (15\%), was $43 \%$. They concluded that a single hCG-H determination in early pregnancy was more accurate, simpler, and faster than standard hCG tests to predict a pregnancy outcome.

Cole reported on a series of 127 early pregnancies (81 normal term pregnancies, 18 miscarriages, and 28 biochemical pregnancies) who underwent urine hCG-H testing ${ }^{12}$. They found that hCG-H urine levels were significantly lower in failing pregnancies when compared to ongoing pregnancies.

Sasaki et al assessed whether the proportion of H-hCG produced at the time of implantation could predict pregnancy success in naturally conceived gestations ${ }^{22}$. They recruited 110 women who were attempting to achieve spontaneous pregnancy. Sixtytwo women became pregnant (42 of them achieved full-term gestation and 20 were not evolutionary gestations). They observed that the ratio of hCG-H/total hCG in urine on the day of implantation (the day with $\mathrm{hCG}>1$ $\mathrm{mUI} / \mathrm{mL}$ ) was associated with the pregnancy outcome. This ratio was $>50 \%$ in all 42 pregnancies that came to term, but only in $35 \%$ of pregnancies that ended in miscarriage. They concluded that proportions of $\mathrm{H}-\mathrm{hCG} /$ total $\mathrm{hCG}>50 \%$ are required for the correct growth and invasion of cytotrophoblast at the time of implantation and a low proportion of H-hCG at the time of implantation may predict pregnancy failure.

More recently, Brady et al reported on a series of 115 women who became pregnant after IFV with blastocyst transfer ${ }^{23}$. They measured serum hCG-H measurement at eleven days after transfer, in order to assess whether hCG-H could predict the pregnancy outcome. Eighty-five pregnancies continued and thirty were failed pregnancies. These authors found that mean hCG-H and its ratio to total hCG were significantly higher in ongoing pregnancies as compared to failed pregnancies. However, hCG-H was not a better predictor than total hCG.

Our results match these studies inasmuch as hCG-H can predict pregnancy outcomes. However, we observed that the hCG-H/total hCG ratio is not a good predictor. This latter finding might be explained by the fact that we focused on women with first-trimester threatened abortion and viable pregnancy at the time of presentation, in naturally conceived pregnancies.

An interesting question to consider is whether these findings could be related to the fact that an actual miscarriage has already happened. However, in our sub-analysis that included only cases with living embryos at the time of clinical presentation, we observe similar results to those for the whole series. This certainly means that early placentas from living embryos in cases of threatened abortion that end in miscarriage release significantly lower amounts of both total hCG and hCG-H, indicating a potential maldevelopment of early sincytotrophoblast that could explain pregnancy outcome.

We think our data might be clinically valuable since we provide new information related to a specific clinical setting. However, we acknowledge that our study has several limitations.

Such limitations include a small sample size. This means that our results should be regarded as preliminary in nature. On the other hand, we observed a miscarriage rate in women with a living embryo as high as $35 \%$ (20 cases out of 57 ), which is substantially higher than expected. Therefore, a potential bias might exist in our study. In addition, we did not perform sample size calculation, so we do not know the statistical power of our findings.

A single hCG-H determination is helpful in predicting pregnancy outcomes in women with first-trimester threatened abortion and viable or potentially viable pregnancy at the time of presentation. hCG-H is not a better predictor than total hCG. However, as we consider our data to be preliminary, further studies are needed to confirm these findings.

\section{Acknowledgements}

We would like to thank Dr Amaia Zornoza for helping to carry out the study at the CHN. 


\section{REFERENCES}

1. REGan L, RaI R. Epidemiology and the medical causes of miscarriage. Bailliere's Best Pract Res Clin Obstet Gynaecol 2000; 14: 839-854. https://doi.org/10.1053/beog.2000.0123

2. Sociedad Española de Ginecología y Obstetricia. Aborto espontáneo. Madrid: SEGO, 2010. https://blog.utp.edu.co/maternoinfantil/files/2012/04/Aborto-espontaneo-1-trimestre.pdf

3. Doubilet PM, Benson CB, Bourne T, Blaivas M. Diagnostic criteria for nonviable pregnancy early in the first trimester. N Engl J Med 2013; 369: 1443-1451. https://doi.org/10.1056/nejmra1302417

4. Krishnamoorthy $\mathrm{K}$, Mouhayar Y. Diagnosis and management of pregnancy loss. Handb Gynecol 2017; 1: 305-313. https://doi. org/10.1007/978-3-319-17798-4_31

5. Norwitz ER, Schust DJ, Fisher SJ. Implantation and the survival of early pregnancy. $\mathrm{N}$ Engl J Med 2001; 345: 1400-1408. https://doi. org/10.1056/nejmra000763

6. Pinar MH, Gibbins K, He M, Kostadinov S, Silver R. Early pregnancy losses: review of nomenclature, histopathology, and possible etiologies. Fetal Pediatr Pathol 2018; 37: 191-209. https://doi.org/10.1080/15513815.2018.14557 75

7. Hasan R, Baird DD, Herring AH, Olshan AF, Jonsson Funk ML, HaRTMann KE. Patterns and predictors of vaginal bleeding in the first trimester of pregnancy. Ann Epidemiol 2010.; 20: 524-531. https://doi.org/10.1016/j.annepidem.2010.02.006

8. Mol F, Ankum WM, Hajenius PJ, Opmeer BC, van Mello NM, Coomarasamy A et al. Diagnostic value of serum hCG on the outcome of pregnancy of unknown location: a systematic review and meta-analysis. Hum Reprod Update 2012; 18: 603-617. https://doi.org/10.1093/ humupd/dms035

9. Faranesh R, Ben-David Y. [Persistent low levels of human chorionic gonadotropin in the serum: investigation and management]. Harefuah 2009; 148: 101-103.

10. Sutton-Riley JM, Khanlian SA, Byrn FW, Cole LA. A single serum test for measuring early pregnancy outcome with high predictive value. Clin Biochem 2006; 39: 682-687. https:// doi.org/10.1016/j.clinbiochem.2006.03.020

11. Chung K, Allen R. The use of serial human chorionic gonadotropin levels to establish a viable or a nonviable pregnancy. Semin Reprod Med 2008; 26: 383-390. https://doi. org/10.1055/s-0028-1087104
12. Cole LA. Hyperglycosylated hCG and pregnancy failures. J Reprod Immunol 2012; 93 : 119-122.

13. Cole LA. Hyperglycosylated hCG, a review. Placenta 2010; 31: 653-164. 14.

14. Cole LA, Khanlian SA. Hyperglycosylated hCG: a variant with separate biological functions to regular hCG. Mol Cell Endocrinol 2007; 260-262: 228-236. https://doi. org/10.1016/j.mce.2006.03.047

15. ColE LA. The O-linked oligosaccharide structures are striking different on pregnancy and choriocarcinoma HCG. J Clin Endocrinol Metab 1987; 65: 811-813. https://doi.org/10.1210/ jcem-65-4-811

16. Cole LA, Butler SA. B152 anti-hyperglycosylated human chorionic gonadotropin free $\beta$-Subunit. A new, possible treatment for cancer. J Reprod Med 2015; 60: 13-20. J Reprod Med;60:13-20.

17. Kovalevskaya G, Kakuma T, Schlatterer J, O'ConNOR JF. Hyperglycosylated HCG expression in pregnancy: cellular origin and clinical applications. Mol Cell Endocrinol 2007; 260-262: 237-243. https://doi.org/10.1016/j. mce.2006.02.021

18. Ko G, Genbace O, Fisher SJ, Caceres E. Trophoblast origin of hCG isoforms : cytotrophoblasts are the primary source of choriocarcinoma-like hCG. Mol Cell Endocrinol 2002; 194: 147-155. https://doi.org/10.1016/s03037207(02)00135-1

19. Handschun K, Guibourdenche J, Tsatsaris V, Guesnon M, Laurendeau I, Evain-Brion D et al. Human chorionic gonadotropin produced by the invasive trophoblast but not the villous trophoblast promotes cell invasion and is down-regulated by peroxisome proliferator-activated receptor- $\gamma$. Endocrinology 2007; 148: 5011-5019. https://doi.org/10.1210/ en.2007-0286

20. Evans J. Hyperglycosylated hCG: a unique human implantation and invasion factor. Am J Reprod Immunol 2016; 75: 333-340. https:// doi.org/10.1111/aji.12459

21. Szczerba A, Bialas P, Pięta PP, Jankowska A. hCG - related molecules and their measurement. Ginekol Pol 2016; 87: 65-70. https://doi. org/10.17772/gp/60981

22. SASAKI Y, LADNER DG, Cole LA. Hyperglycosylated human chorionic gonadotropin and the source of pregnancy failures. Fertil Steril 2008; 89: 1781-1786. https://doi.org/10.1016/j. fertnstert.2007.03.010

23. Brady PC, Farland L V., Racowsky C, Ginsburg ES. Hyperglycosylated human chorionic gonadotropin as a predictor of ongoing pregnancy. 
Am J Obstet Gynecol 2019;222: 68.e1-68.e12. https://doi.org/10.1016/j.ajog.2019.08.004

24. Kovalevskaya G, Birken S, KaKuma T, O'Connor JF. Early pregnancy human chorionic gonadotropin (hCG) isoforms measured by an immunometric assay for choriocarcinoma-like hCG. J Endocrinol 1999; 161: 99-106. https:// doi.org/10.1677/joe.0.1610099

25. Evans J, Salamonsen LA, Menkhorst E, Dimitriadis E. Dynamic changes in hyperglycosylated human chorionic gonadotrophin throughout the first trimester of pregnancy and its role in early placentation. Hum Reprod 2015; 30: 10291038. https://doi.org/10.1093/humrep/dev016

26. Guibourdenche J, Handschuh $\mathrm{K}$, Tsatsaris V, Gerbaud P, Leguy MC, Muller F et al. Hyperglycosylated hCG is a marker of early human trophoblast invasion. J Clin Endocrinol Metab 2010; 95: 240-244. ttps://doi.org/10.1210/ jc. $2010-0138$

27. Berndt S, Blacher S, Munaut C, Detilleux J, D'hauterive SP, Huhtaniemi I et al. Hyperglycosylated human chorionic gonadotropin stimulates angiogenesis through TGF- $\beta$ receptor activation. FASEB J. 2013; 27: 1309-1321. https://doi.org/10.1096/fj.12-213686

28. Kovalevskaya G, Birken S, Kakuma T, OzaKi N, SAUER M, LindHeim S et al. Differential expression of human chorionic gonadotropin (hCG) glycosylation isoforms in failing and continuing pregnancies: Preliminary characterization of the hyperglycosylated hCG epitope. J Endocrinol 2002; 172: 497-506. https://doi.org/10.1677/joe.0.1720497 
